\title{
Exploring the Dynamics of Large-Scale Biochemical Networks: A Computational Perspective
}

\author{
Ralf Steuer*,a,b \\ ${ }^{a}$ Humboldt-University of Berlin, Institute for Theoretical Biology (ITB), Invalidenstr. 43, 10115 Berlin, Germany \\ ${ }^{b}$ Manchester Interdisciplinary Biocentre, The University of Manchester, 131 Princess Street, M1 7DN, Manchester, UK
}

\begin{abstract}
The complexity of even comparatively simple biochemical systems necessitates a computational description to explore and eventually understand the dynamics emerging from the underlying networks of cellular interactions. Within this contribution, several aspects relating to a computational description of large-scale biochemical networks are discussed. Topics range from a brief description of the rationales for computational modeling to the utilization of Monte Carlo methods to explore dynamic properties of biochemical networks. The main focus is to outline a path towards the construction of large-scale kinetic models of metabolic networks in the face of incomplete and uncertain knowledge of kinetic parameters. It is argued that a combination of phenotypic data, large-scale measurements, heuristic assumptions about generic rate equations, together with appropriate numerical schemes, allows for a fast and efficient way to explore the dynamic properties of biochemical networks. In this respect, several recently proposed strategies that are based on Monte Carlo methods are an important step towards large-scale kinetic models of cellular metabolism.
\end{abstract}

Keywords: Systems biology, dynamical modeling, metabolism.

\section{INTRODUCTION}

All biological systems, from single pathways to multicellular organisms, continuously respond to, change, adapt, and regulate their internal biochemical states. However, cellular interaction maps are usually depicted as static networks, such that a compound or entity of interest is connected or linked (borrowing concepts from graph theory) to one or more other compounds or entities. Although usually owed to constraints in visualization, such a static depiction provides a misleading impression of cellular interactions and organization. Cellular interactions are dynamic - they are characterized by a certain strength at a certain time, which might change according to cellular circumstances. For example, it is known that allegedly interacting proteins are not necessarily expressed concurrently at the same time or in the same tissue. Similar, metabolism constantly reshapes its fluxes in response to available nutrients and other environmental or intracellular factors, resulting in a temporal compartmentation of metabolic processes.

Unfortunately, while the topology of cellular interactions is increasingly known, detailed information about the dynamics of cellular interactions, their strength in different tissue and at different spatial and temporal locations, is only scarcely available. Nonetheless, a growing number of studies emerge that aim to describe the kinetics and temporal behavior of biological interactions - aiming to develop dynamic mathematical models of cellular processes. Current mathematical models are not only confined to classic

*Address correspondence to this author at the Humboldt-University of Berlin, Institute for Theoretical Biology (ITB), Invalidenstr. 43, 10115 Berlin, Germany; Tel: +49 (0)30 2093 9119; Fax: +49 (0)30 2093 8801;

E-mail: ralf.steuer@manchester.ac.uk instances of temporal cellular regulation, such as circadian clocks and other cellular rhythms [1], pattern formation and development [2,3] or small signalling circuits [4], but increasingly aim at large-scale metabolic and regulatory networks [5-10].

This review seeks to describe and summarize recent efforts to characterize the dynamics of biological and biochemical systems. Our focus is the computational perspective, namely the construction of large-scale kinetic models to describe biological processes. The manuscript is organized as follows: The first section provides a brief description of the concepts and history of mathematical modeling of biochemical networks. In the second section, the key steps to translate a given pathway diagram into a kinetic model are discussed. In this respect, of particular interest are heuristic and approximative methods that allow to deal with incomplete and uncertain data, such as methods based on Monte Carlo analysis. The next section is focussed on the computational description of metabolic networks. It is shown that some functional regularities of enzyme-catalyzed reactions allows for an efficient computational description and simulation of metabolic networks. Subsequently, a topdown strategy for the analysis and simulation of metabolic networks is presented which may serve as a stepping stone for the construction of genome-scale kinetic models.

\section{MODELING OF BIOCHEMICAL SYSTEMS}

Mathematical modeling has a long history in the biochemical and biological sciences, tracing back at least to the description of simple enzyme kinetics by Henri, Haldane, Michaelis and Menten. Probably the first computational model - in the sense the term is understood today - of a biochemical process was published by Britton Chance in 1943 [11], who used a mechanical differential analyzer to 
solve the equations for the behavior of a simple enzymatic system. The pioneering work of Chance was taken up and continued in the 1950s and 1960s, resulting in metabolic models of increasing complexity and size, see Garfinkel et al. [12] for an early review. Not surprisingly, these early simulations were severely limited by inadequate computer hardware and the lack of appropriate algorithms to deal with the stiffness of differential equations describing the dynamics of biochemical systems [13]. Only the rather recent advances in computational feasibility have allowed to perform large-scale simulation routinely - with a renewed interest in computational methods triggered by the advent of Systems Biology [14].

However, despite the long history and the recent surge in applications, several fundamental questions relating to computational modeling as a credible research tool are not resolved. There are no clear criteria what properties constitute a good model. What standards can be conjured up to separate good and bad models? And, once constructed, what can be learned from a good model? As put by J. E. Bailey, "modeling is relatively meaningless without explicit definition, at the outset, of its purpose" [15]. Clearly, to answer such questions is no trivial task. For example, recently the capability to successfully em predict cellular outcomes has been suggested as the ultimate hallmark of modeling [16]. However, the ability to predict does not entail the ability to explain. As pointed out by Casti [17], the Ptolemaic geocentric system of astronomy was exceedingly predictive in its time and initially also outperforming the Copernican system within the available standards of observational accuracy. Nonetheless, the Ptolemaic system was woefully inadequate to explain the movements of planets. Similar problems arise for almost any simple and straightforward criterion to judge the quality and standards of model construction.

Nonetheless, computational modeling has its firm place in current molecular biology. In practice, modeling of biochemical phenomena is usually performed with one or both of the following two rationales in mind: First, models are a method of representation: As noted by Yuri Lazebnik in his iconic article [18], the language used by biologists for communications is not unlike that used by stock market analysts. Both disciplines use vague expressions and avoid clear predictions. To overcome the pitfalls of imprecise verbal communication, the utilization of a mathematical formalism may provide a suitable language to describe observed functional relationships. For example, a reasonably precise and unambiguous description of the functional form of an observed interaction, rather than a vague verbal expression, is a prerequisite for other researcher to contrast a reported result with their own observation. Second, models are a method of deduction: Once the individual interactions are described in an appropriate formal language, mathematical theory and computational methods provide a tremendously powerful set of tools to explore the emergent properties of biochemical networks. Computational models provide a formal framework in which experimental observations can be understood.

In this sense, modeling of biochemical systems, as understood within this article, follows the relation described by Casti [17] and is depicted in Fig. (1). The modeling process provides the appropriate encoding operations to express biological interactions in a formal mathematical language. The encoding of a biological systems into a mathematical framework then allows for a formal interrogation of the systems behavior, utilizing set of rules and computational methods (theorems). The inferred properties of the formal system (the model) become predictions about the biological or biochemical system. The modeler is mainly concerned with the encoding/decoding relations that translate back and forth between the biological and the computational world, whereas specialists in both fields develop the experimental and computational methods that drive progress in either realm. According to this view, mathematical and computational modeling epitomizes one of the the paradigms of systems biology: Mathematical modeling is a systematic and ordered way to describe our current knowledge of biological processes $[15,19,20]$. It allows to address the emergent properties of networks of interactions - properties that are neither accessible by a detailed exploration of the properties of the constituents of the system, nor by intuitive reasoning about how these constituents interact. However, it should be noted that the shift in paradigm often attributed to systems biology, the shift from an interest in properties of constituents to an interest in properties of systems, is not so new after all. No later than 1977, David Garfinkel already cites a remark made by the chemical engineer L. E. Scriven: "Were a chemical engineer to capture a strange factory, the last thing he would think of would be to use a steamroller and then separate the wreckage by sedimentation. Nor would he freeze the factory and saw it up for analysis" [13]. Similar, the current foundations of kinetic modeling of biochemical systems have been laid already almost 40 years ago, most notably

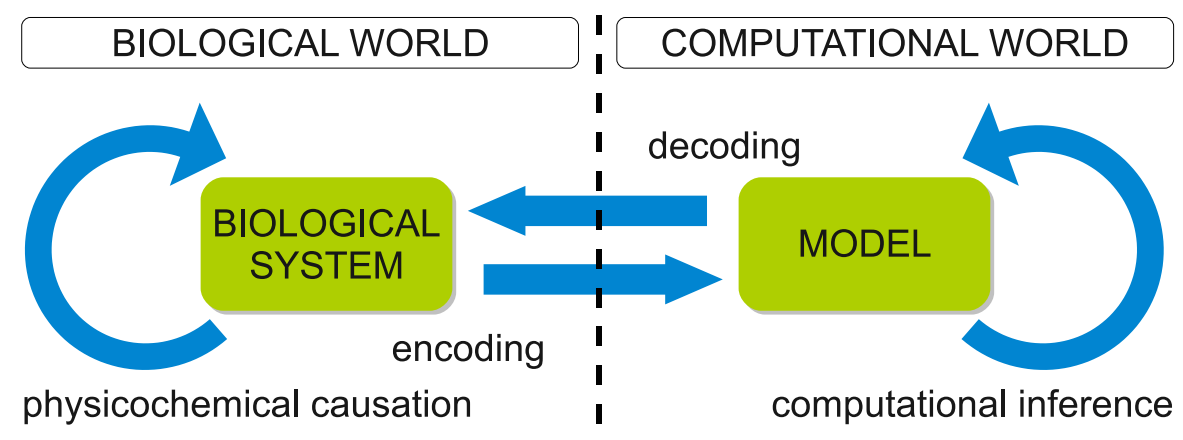

Fig. (1). The modeling relation according to Casti [17]. The modeling process is based on the encoding and decoding relations that translate between the biological and the mathematical world. 
with the formulation of Biochemical Systems Theory (BST) by M. Savageau [21, 22] and later Metabolic Control Analysis [23, 24].

\section{FROM TOPOLOGY TO DYNAMICS}

Obviously, there exists no single mathematical or computational technique or framework that is able to comprehensively cover all aspects of a biological system. Rather, computational modeling has many facets and covers a broad range of methodologies and applications [20]. Current computational models of biological systems include purely topological [25] and stoichiometric descriptions [2628], as well as Boolean models [29, 30], models based on ordinary differential equations [20], spatial models [31], stochastic models [32-34], Petri nets [35, 36], and combinations thereof, just to name a few. Even when focusing entirely on a description based on ordinary deterministic differential equations (ODEs) - arguably still the most prevalent method for modeling biological systems we are faced with a rich diversity in formal descriptions and approximative schemes. See also Steuer and Junker [20] for a more detailed discussion. While in the following our focus is almost entirely on models based on ODEs, most issues are likewise applicable to other formal computational frameworks.

In principle, the construction of a computational model is rather straightforward. Given a biological system of interest, one needs to run through a series of simple and defined steps until the final model is obtained.

Specifically: (i) The building blocks of the system of interest must be assembled, that is, a list of all participating compounds and their interactions must be compiled. (ii)
Each interaction has to be assigned to a specific functional form, given by a rate equation or other mathematical rule that specifies the nature of the interaction. (iii) Next, a quantitative value has to be assigned to each kinetic parameter. The values of kinetic parameters are obtained from the primary literature, dedicated databases, or specifically determined experimentally for the system at hand. Alternatively, parameters can be estimated from global measurements and time series data [37, 38]. (iv) Finally, the fully parameterized model can be interrogated using the sophisticated computational machineries developed in the past decades. The model needs to be validated by additional data, not already used in its construction. Novel experiments can be formulated based on predictions of the model. The entire process is exemplified in Fig. (2).

However, in practice, a number of obstacles hamper the straightforward construction of computational models. Only rarely the interaction topology of a biological phenomenon is comprehensively known. Likewise, the functional form of biological interactions is often difficult to assess. In many cases, functional interactions are not direct but mediated by additional compounds or processes which are not explicitly accounted for in the model - resulting in potentially arbitrarily complex interaction functions. Even for direct interactions, the respective interaction functions may be highly nonlinear. In particular for interactions involving multiple components, the precise functional form usually cannot be inferred from first principles and is difficult to map experimentally [39]. The most significant obstacle, however, is still the lack of reliable quantitative data with sufficient accuracy to animate computational models. Despite the torrent of data accompanying the advances in 'omics' technologies, these data are often not suitable for

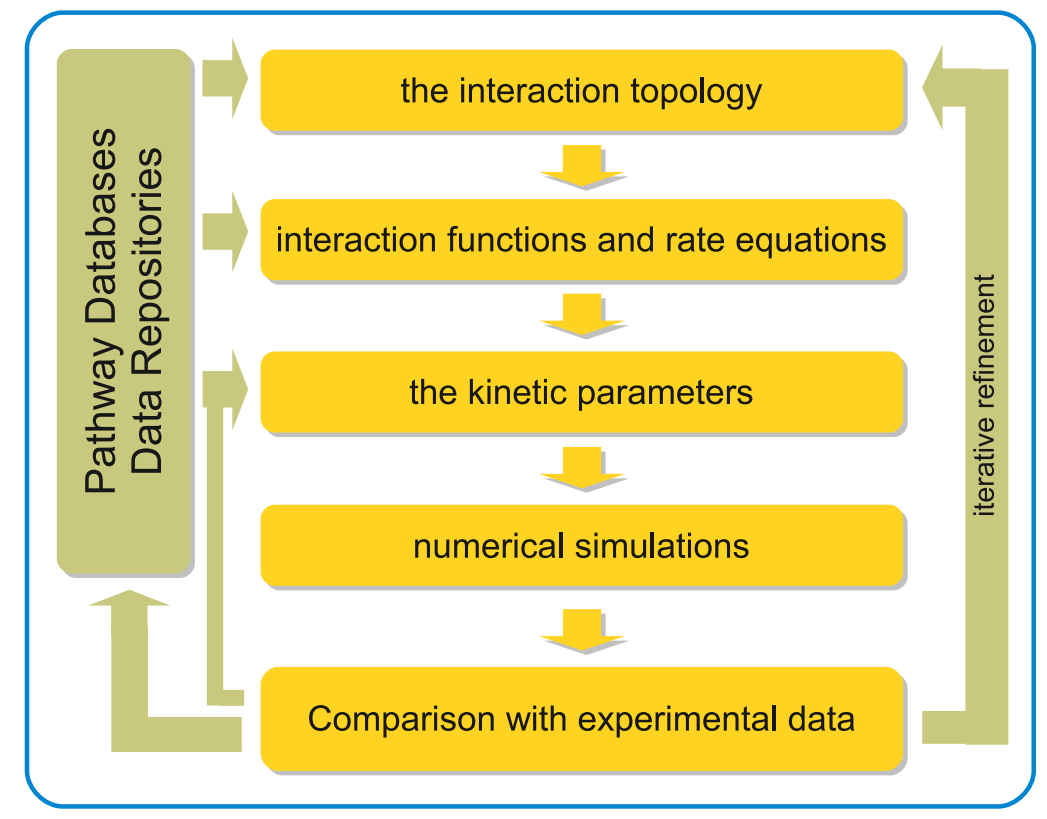

Fig. (2). In principle a computational model of a biochemical network is easily constructed: First, the interaction topology of the model is assembled, either in the form of a list of all interactions or as a pathway diagram. These 'building blocks' are usually extracted from available pathway repositories and databases. Second, each interaction is assigned to a specific functional form and the network is assembled into a system of differential equations (or any other suitable mathematical representation). Third, each kinetic parameter is assigned to a quantitative value obtained either from direct experiments, the primary literature, or data repositories. Finally, the model can be evaluated using computational methods and compared to experimentally observed timecourses or phenotypic data. The process is repeated iteratively. 
model construction. Either, as for most data obtained by large scale methods, measurements are only qualitative or at best semi-quantitative. Or, the data do not cover the specific requirements of interest, for example in terms of timeresolution, temperature regimes, sub-cellular resolution or other constraints. As yet, parameterization of kinetic models remains a laborious process, integrating data from diverse sources - often associated with considerable compromise.

Given these profound obstacles in model construction, it is not surprising that the main challenge of computational modeling is to deal with uncertain and only partially available kinetic information. Several strategies have been suggested, each with specific assets and drawbacks. At a basic level, three broad categories can be distinguished: The first category consists of strategies that seek to describe the dynamics of biochemical systems without any recourse on kinetic parameters. Examples include logical models, models using parameter-free threshold functions, as well as models based on Boolean dynamics [30, 40-42]. However, the dynamics found in such models is often qualitatively different from results obtained from a description by conventional chemical kinetics. Furthermore, some variants of parameter-free dynamics are not genuinely parameter-free but implicitly assume heuristic global parameters which are set equal for all interaction functions. The second category consists of methods that seek to derive stringent statements on the dynamics of biochemical systems, solely based on knowledge of its topology or structure. The most prominent examples are chemical reaction network theory (CRNT), developed by Horn, Jackson, and Feinberg [43-45], and stoichiometric network analysis (SNA), developed by B. L. Clarke [46]. Both approaches provide an algebraic framework to relate aspects of network structure to various kinds of dynamics in a systematic way and only rely on weak assumptions about kinetic parameters. The last category consists of Monte Carlo methods to evaluate the possible behavior of biochemical networks and explicitly account for uncertainty in network structure and parameters [9, 16, 47-56]. Monte Carlo methods offer the advantage that they do not necessarily rely on simplifying assumption, but rather aim to translate uncertainties in input parameters into uncertainties or probabilities of output quantities. However, while conceptually often straightforward, Monte Carlo methods are usually computationally expensive and require extensive multiple numerical evaluations of a pathway of interest. In the next section, Monte Carlo sampling as a tool to describe the dynamics of metabolic networks is discussed.

\section{THE DYNAMICS OF METABOLIC NETWORKS}

Cellular metabolism - the biochemical interconversion of small molecules catalyzed by enzymes - is an important determinant of cellular physiology and of relevance for many biotechnological and medical applications. In contrast to other areas of cellular regulation, computational modelling has played a valuable role in understanding the functioning of metabolic pathways for many decades. Metabolic systems are particularly accessible to a systematic computational approach for a number of reasons: First, the function of biochemical pathways is usually exerted by mass-transfer. This fact makes it comparatively easy resolve the topological organization, for example by isotopic labeling techniques that trace the passage of a compound through the pathway.
Consequently, the topology of metabolic networks is often reasonably well known, at least as compared to the topology of other networks of cellular regulation. Second, the mechanisms and kinetics of single enzymes have been extensively studied for more than a century. The mode of action of single enzymes, as the building blocks of largescale computational models, are therefore reasonably well understood and are accessible by a large number of experimental techniques. Third, most components and pathways of a metabolic network have a well-defined functionality, for example to provide cellular energy in the form of ATP or to provide building blocks for cellular growth. Taken together, a known topology, a known functional form of most interactions, and a clear-cut functionality establish a rewarding basis for the translation of a biological network into a computational model.

Given the opportune properties of metabolic networks, it is not surprising that many current computational studies on cellular metabolism already aim at a rather large-scale description and are often reasonably predictive [20, 27, 57, 58]. However, most current work is focussed on flux-balance analysis (FBA), a methodology that requires only knowledge of the stoichiometry of the network. While FBA is a tremendously powerful tool to assess the large-scale properties of metabolic networks, additional methods are needed to describe the dynamic properties of such networks. In this respect, there is increasing interest to bridge the gap between the already successful topological description and the scarcity of dynamic models [6, 7, 9, 48-50, 59-61]. The aim of most of these studies is to unite the advantages of FBA, its computational efficiency and predictive power, with a more detailed description of the dynamics of the system.

In this respect, a promising approach is to exploit the functional regularities of enzymatic reactions to construct kinetic first-draft models, followed by a Monte Carlo evaluation of model properties. Rather than building upon detailed knowledge of the kinetics of each individual reaction step, the kinetic description may start with heuristic assumptions about generic reaction mechanisms. Enzyme kinetic rate laws are usually derived from mechanistic descriptions, based on a number of simplifying assumptions such as quasi-steady state or rapid equilibrium assumptions [20]. Although the resulting detailed functional forms might considerable differ and depend on the specific minutiae of enzymes and binding mechanisms, most enzymatic reaction equations share several common features: (i) The flux through a reaction depends on the available substrates of the reaction. If one or more substrates are not available, the reaction does not proceed. Usually, the flux continuously increases with increasing substrate concentrations, although exceptions of this rule exist due to saturation or allosteric regulation. (ii) As the amount of available enzyme is limited, there exists a region where a further increase in substrate availability does not result in an increased flux. Enzymecatalyzed reactions exhibit saturation. (iii) All chemical reactions are reversible. The direction of a reaction is determined by the change of free energy. In particular, if substrates and products are in thermodynamic equilibrium, the net flux is zero. 
A large number of approximate functional forms for generic reaction kinetics has been suggested and compared in the literature [61-66]. However, most simple choices, such as as mass-action, power-law or lin-log kinetics, violate one or more of the requirements stated above. For a generic reaction mechanism that interconverts $n_{s}$ substrates $\mathbf{A}$ into $n_{p}$ products $\mathbf{B}$,

$$
A_{1}+A_{2}+\ldots+A_{n_{s}} \leftrightarrow B_{1}+B_{2}+\ldots+B_{n_{p}}
$$

a reasonable functional form, in compliance with basic biochemical and thermodynamic requirements, is given by the expression

$$
r(\mathbf{A}, \mathbf{B})=\frac{V_{M}}{\prod_{i=1}^{n_{s}} K_{M_{i}}} \frac{\left(\prod_{i=1}^{n_{s}} A_{i}-\prod_{j=1}^{n_{p}} \frac{B_{j}}{K_{\text {eq }}}\right)}{F\left(\mathbf{A}, \mathbf{B}, \mathbf{K}_{M}\right)} .
$$

Within this heuristic Michael-Menten-type equation (1) the vectors $\mathbf{A}=\left\{A_{1}, \ldots, A_{n_{s}}\right\}$ and $\mathbf{B}=\left\{B_{1}, \ldots, B_{n_{p}}\right\}$ denote the concentrations of substrates and products, respectively; $K_{\text {eq }}$ denotes the thermodynamic equilibrium constant; $V_{M}$ denotes a maximal reaction velocity; and $F\left(\mathbf{A}, \mathbf{B}, \mathbf{K}_{M}\right)$ denotes a positive polynomial that depends on kinetic parameters $\mathbf{K}_{M}$. Additional factors that account for allosteric regulation may be included, either as a multiplicative term corresponding to a modulation of the maximal reaction velocity $V_{M}$, or, as an additive term on the polynomial $F$, corresponding to competitive inhibition.

The generic rate equation described in Eq. (1) is reversible, with a net-flux $r=r^{+}-r^{-}$that is given by the difference between the forward and backward direction. The direction of the reaction is determined by the ratio

$$
\frac{r^{-}}{r^{+}}=\frac{\prod_{j=1}^{n_{p}} B_{j}}{\prod_{i=1}^{n_{s}} A_{i}} \frac{1}{K_{\mathrm{eq}}}=: \frac{\Gamma}{K_{\mathrm{eq}}},
$$

where $\Gamma$ denotes the mass-action ratio. To emphasize the distinction between thermodynamic and kinetic properties, Eq. (1) can be expressed as

$$
r(\mathbf{A}, \mathbf{B})=\underbrace{\frac{V_{M} \prod_{i=1}^{n_{s}} \frac{A_{i}}{K_{M_{i}}}}{F\left(\mathbf{A}, \mathbf{B}, \mathbf{K}_{M}\right)}}_{=r^{+}(\mathbf{A}, \mathbf{B})}\left(1-\frac{\Gamma}{K_{\mathrm{eq}}}\right) .
$$

A number of options are available to specify the detailed functional form of $F\left(\mathbf{S}, \mathbf{P}, \mathbf{K}_{M}\right)$. Possible choices are discussed in $[61,62,66]$ and are usually based on the assumption of a generic random-order binding mechanism of substrates and products.

Once the functional form of each reaction equation is assigned, the metabolic pathway or network is translated into a system of differential equations, following the steps shown in Fig. (2). The system of ODEs can then be written in the compact form

$$
\frac{\mathrm{d} \mathbf{S}}{\mathrm{d} t}=\mathbf{N} \cdot \mathbf{r}(\mathbf{S})
$$

where $\mathbf{N}$ denotes the stoichiometric matrix and $\mathbf{S}$ the vector of all substrates and products within the network.

As most of the kinetic parameters are usually unknown, an analysis of Eq. (4) may commence with a Monte Carlo strategy to obtain information about the typical or possible dynamic behavior of the network. Sampling the parameters is not straightforward, but must incorporate a number of physicochemical constraints to ensure plausibility of the ensemble of models. In particular, assuming a known stoichiometry, three types of parameter values are required to animate the rate equations given by Eq. (1): (i) The equilibrium constant $K_{\text {eq }}$ : The value $K_{\text {eq }}$ is a physicochemical quantity and entirely determined by the change in standard Gibbs free energy of the reaction. While not dependent on the specific organism or cell type, the thermodynamic equilibrium is sensitive to a number of intracellular parameters, such as temperature. While a detailed experimental quantification is often not available, a number of algorithms exists that allow for a reasonable computational approximation [67-71]. Approximative values of $K_{\text {eq }}$ for each reaction can therefore be assigned even for reasonably large networks. (ii) The affinity or MichaelisMenten constants $\mathbf{K}_{M}$ : Although the experimental estimation of Michaelis-Menten constants is possible and rather straightforward, such estimates are usually done on an individual basis and large-scale automation is not possible. An increasing number of repositories $[72,73]$ and automated retrieval systems [74] exist. Nonetheless uncertainty in affinity constants is certainly one of the major constraints in kinetic modeling. Furthermore, values provided in data repositories are often not accompanied by the raw data, along with the methods and the functional forms used for their estimation, which significantly hampers their reutilization in different conditions. (iii) The maximal reaction velocities $V_{M}$ : The parameters representing the catalytic activity and maximal reaction velocity are arguably the most difficult parameters to assign on a large-scale level. Though parallel measurements are possible [75], the values are dependent on enzyme expression - and may thus change rapidly and significantly with respect to different conditions. Also post-translational modification mechanisms, such as the deactivation of enzymes by phosphorylation, alter the effective values of $V_{M}$. While in the long run, enzyme concentrations and their respective activity will be kinetic variables within integrated models, rather than kinetic parameters, most current models treat enzyme concentrations and activity as a constant quantities - thereby making the model highly dependent on experimental circumstances.

Utilizing a Monte Carlo strategy and given the considerations above, the kinetic parameters may be sampled according to the following rules: The equilibrium constant 
$K_{\text {eq }}$ are globally assigned to best-guess values, as obtained from large-scale approximation of thermodynamic properties. The affinity or Michaelis-Menten constants $\mathbf{K}_{M}$ are sampled randomly in intervals $\left[K_{M}^{\min }, K_{M}^{\max }\right]$, with either global boundaries common for each parameter or boundaries assigned individually for each parameter. The choice of the boundaries may be guided by the distribution of available values in large-scale repositories. The intervals may further be refined if similar reactions have been experimentally characterized in related organisms. Within the assigned interval, the values may either be sampled from a uniform distribution, or from a distribution that is, for example, constructed according to the entirety of kinetic parameters available in large-scale repositories [76]. In general, different variants for the sampling strategy should be tested - it must be ascertained that reported results are not an artifact of a particular strategy, but are robust against slight variations in the methodology. The sampling of the maximal reaction velocities may follow similar rules as for the affinity constants. However, independent sampling of velocity parameters for each reaction may easily lead to unphysiological bottlenecks in the network. Alternatively, the maximal reaction velocities may be assigned such that they exceed a minimal value - with the minimal value for each reaction chosen according to a previously determined steady-state flux distribution. A crude first-guess approach is to globally assign the maximal velocities to rather high values, such that they exceed any expected flux value. Such a strategy is warranted by the observation that most reaction do not operate close to saturation and most enzyme activities are in large surplus relative to the observed in vivo fluxes [77]. More refined methods to chose appropriate reaction velocities are discussed below.

For each sampled instance of the parameters the model properties are evaluated. While the ensemble will not necessarily pinpoint the actual behavior of a pathway, evaluation of the ensemble allows us to test for generic or possible behavior and to check the consistency with experimentally observed behavior. In addition to straightforward sampling, the parameter space can also be evaluated using more sophisticated methods, such as a targeted search for parameter region corresponding to specific behavior [78]. The evaluation may be further refined using partial information on rate laws and kinetic parameters, as well as model selection based on available phenotypic data [54].

\section{STRUCTURAL KINETIC MODELING}

An alternative strategy that aims to avoid some of the difficulties and pitfalls associated with determining the appropriate distributions for parameter-sampling was suggested recently [20, 48, 51]. Instead of using a classic bottom-up approach of model construction, we propose to implement a top-down approach that is based on experimentally available physiological data. In particular, while most kinetic parameters are not directly accessible using high-throughput techniques, great strides have been made in the large-scale characterization of metabolic systems in terms of their concentrations and fluxes. High-throughput metabolomics and fluxomics studies are now almost standard techniques in the analysis of cellular metabolism [79-85]. Given the recent progress in technical possibilities, the comprehensive quantification of all concentrations and fluxes within a metabolic system is, at least in principle, experimentally feasible $[86,87]$.

The intervals for kinetic parameters may thus be chosen such that the resulting kinetic model is consistent with an experimentally determined metabolic state. Of course, an observed set of concentrations and fluxes does not allow to uniquely identify the complete set of kinetic parameter rather the observed concentrations and fluxes implicitly specify an ensemble of possible models - which is subsequently subject to statistical evaluation. The top-down strategy is illustrated in Fig. (3).

In practice, we assume knowledge of a - not necessarily unique or stable - state that satisfies the steady-state condition $\mathbf{N} \cdot \mathbf{r}^{0}=\mathbf{0}$, where $\mathbf{r}^{0}:=\mathbf{r}\left(\mathbf{S}^{0}\right)$ denotes the stationary flux vector at steady state concentrations $\mathbf{S}^{0}$. The set $\left\{\mathbf{r}^{0}, \mathbf{S}^{0}\right\}$ characterizes the metabolic state of the system.

To construct an ensemble of models consistent with the metabolic state, the parameters are sampled according to the following rules: $(i)$ The equilibrium constants $K_{\text {eq }}$ are chosen as above and, in absence of experimental data, are assigned to values obtained from computational approximations. (ii) The affinity or Michaelis-Menten constants $\mathbf{K}_{M}$ are assigned to (either global or individual) intervals $\left[K_{M}^{\min }, K_{M}^{\max }\right]$. In addition to the criteria given above, interval boundaries can be chosen according to known metabolite concentrations. For example, the interval may be constraint to a 10 or 100 -fold variation around the maximal and minimal concentrations values, using a logarithmic distribution. (iii) Finally, the maximal reaction velocities $V_{M}$ are chosen such that the system is consistent with its metabolic state $\left\{\mathbf{r}^{0}, \mathbf{S}^{0}\right\}$. In particular, with all other parameters assigned, the value of $V_{M}$ for each reaction is uniquely determined. Given the functional form Eq. (1), the parameter $V_{M}$ can be calculated as

$$
V_{M}=\frac{r^{0} \cdot \prod_{i=1}^{n_{s}} K_{M_{i}} \cdot F\left(\mathbf{A}^{0}, \mathbf{B}^{0}, \mathbf{K}_{M}\right)}{\left(\prod A_{i}^{0}-\prod \frac{B_{j}^{0}}{K_{\mathrm{eq}}}\right)}
$$

Utilizing the rules specified above, any set of sampled Michaelis-Menten constants is, by definition, consistent with the metabolic state $\left\{\mathbf{r}^{0}, \mathbf{S}^{0}\right\}$. A subsequent Monte Carlo analysis then explores the dynamic properties associated with this specific metabolic state.

A further drastic reduction of the numerical effort required for the Monte Carlo analysis can be achieved by utilizing a local linear approximation of the system. In fact, an explicit computational simulation of the system is often 


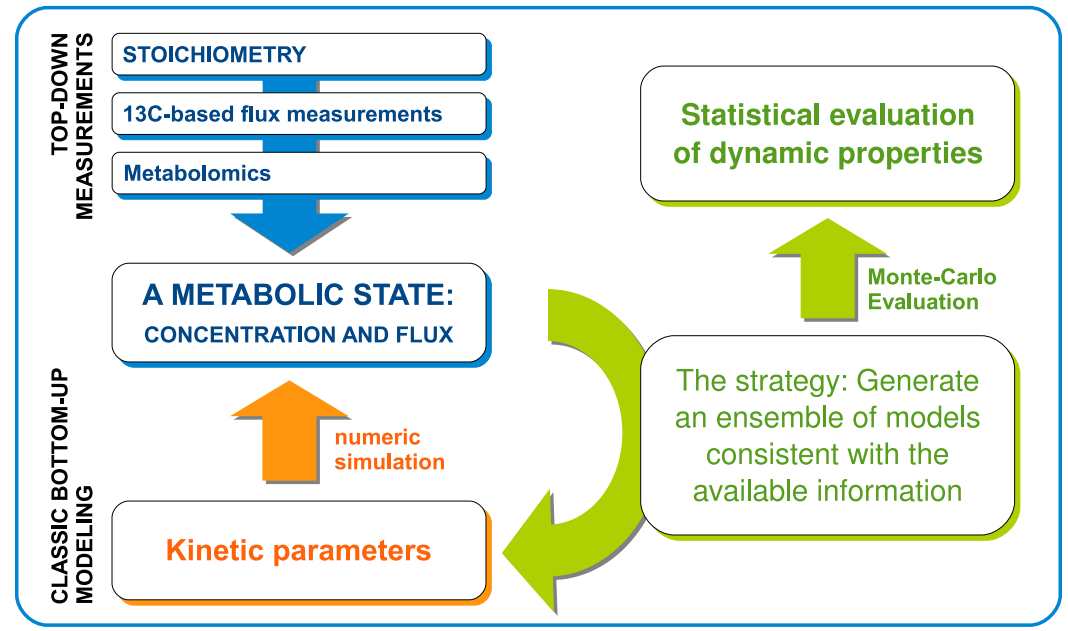

Fig. (3). A top-down strategy to explore the dynamics of large-scale biochemical networks. The basic idea is to generate an ensemble of models that is consistent with available information about the metabolic state of the system. The ensemble of models consistent with the metabolic state then allows for a systematic statistical evaluation of dynamic properties associated with the respective state.

not necessary: Many properties of interest are readily available using only knowledge of the Jacobian matrix $\mathbf{J}$ of the network. The Jacobian matrix determines the stability of a state, the linear response to perturbations, the flux and concentration control coefficients, time hierarchies and several other properties of direct relevance for the dynamics of the system [20, 48, 88]. Formally, the Jacobian matrix $\mathbf{J}$ is obtained by a series expansion of Eq. (4),

$$
\frac{\mathrm{d} \mathbf{S}}{\mathrm{d} t}=\underbrace{\mathbf{N} \cdot \mathbf{r}^{\mathbf{0}}}_{=\mathbf{0}}+\underbrace{\left.\mathbf{N} \cdot \frac{\partial \mathbf{r}}{\partial \mathbf{S}}\right|_{\mathbf{S}^{0}}}_{=: \mathbf{J}}\left(\mathbf{S}-\mathbf{S}^{\mathbf{0}}\right)+\ldots .
$$

The first term in the expansion describes the flux-balance constraint, which provides the foundation for FBA. Going beyond the zero-th order can thus be interpreted as a way to augment FBA with an additional term that takes into account some of the dynamic properties of a given steady-state flux distribution. In particular, utilizing the sampling strategies discussed above, the Jacobian matrix can be estimated by simple matrix multiplication and requires no further computationally expensive simulations. To obtain an expression for the Jacobian matrix, defined as

$$
\mathbf{J}=\left.\mathbf{N} \cdot \frac{\partial \mathbf{r}}{\partial \mathbf{S}}\right|_{\mathbf{S}^{0}},
$$

requires an evaluation of the matrix of partial derivatives at the specified state. In this respect, it is advantageous to rewrite Eq.(7) using a logarithmic representation, such that

$$
\mathbf{J}=\mathbf{N} \cdot \mathbf{D}_{\mathbf{r}^{0}} \cdot \mathbf{Q} \cdot \mathbf{D}_{\mathbf{S}^{0}}^{-1},
$$

where $\mathbf{D}_{\mathbf{r}^{\mathbf{0}}}$ and $\mathbf{D}_{\mathbf{s}^{\mathbf{0}}}$ denote diagonal matrices with elements $\mathbf{r}^{\mathbf{0}}$ and $\mathbf{S}^{\mathbf{0}}$ on the diagonal, respectively; and the matrix $\mathbf{Q}$ with elements $Q_{i j}=\partial \ln r_{i} / \partial \ln S_{j}$ denotes the matrix of logarithmic partial derivatives evaluated at the metabolic state. The elements of $\mathbf{Q}$ are usually dimensionless values that specify the relative saturation of the reaction with respect to its substrates and correspond to the scaled elasticities of metabolic control analysis [20]. Once all matrices within Eq. (8) are specified, the Jacobian matrix can be obtain by matrix multiplication.

To exemplify the advantages provided by a top-down strategy based on logarithmic derivatives, we consider a simple metabolic pathway that consists of two reactions, producing and consuming a single metabolite $S$,

$$
\rightarrow S \rightarrow \text {. }
$$

See also Fig. (4).

The systems of ODEs is given as

$$
\frac{d S}{d t}=\left[\begin{array}{ll}
1 & -1
\end{array}\right]\left[\begin{array}{l}
r_{1} \\
r_{2}
\end{array}\right] \text {. }
$$

We assume a constant influx $r_{1}=c$ and an outflux $r_{2}(S)$ that follows a simple Michaelis-Menten kinetics,

$$
r_{2}(S)=\frac{V_{M} S}{K_{M}+S} .
$$

A classic computational analysis would now be based on the three kinetic parameters of the pathway, the influx $c$, the maximal velocity $V_{M}$, and the Michaelis-Menten parameter $K_{M}$.

However, we are interested in an alternative representation based on the metabolic state, characterized by the two matrices

$$
\mathbf{D}_{\mathbf{r}^{\mathbf{0}}}=\left[\begin{array}{cc}
r^{0} & 0 \\
0 & r^{0}
\end{array}\right] \text { and } \quad \mathbf{D}_{\mathbf{s}^{\mathbf{0}}}=S^{0},
$$

where $r^{0}=r_{1}^{0}=r_{2}^{0}$ denotes the steady state flux. To obtain an expression for the Jacobian matrix, we need to evaluate the elements of the matrix $\mathbf{Q}$,

$$
\mathbf{Q}=\left[\begin{array}{l}
0 \\
\theta
\end{array}\right],
$$




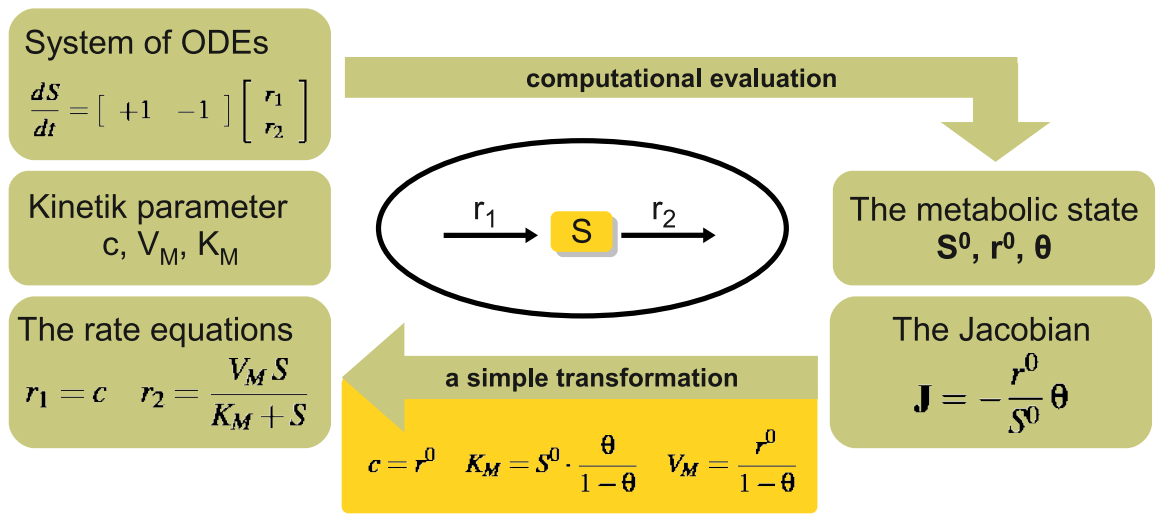

Fig. (4). The description of a biochemical network in terms of a metabolic state provides allows for a fast and efficient evaluation of its dynamic properties. Usually, following the conventions of bottom-up modelling, a set of kinetic parameters gives rise to a metabolic state for example obtained by numerical integration of the corresponding set of differential equations. This transformation is non-unique: Several metabolic states may exist for any given set of parameters (bi- or multistability). Conversely, the metabolic state can be interpreted as a set of parameters to characterize the biochemical system. In this case, the Jacobian can be obtained straightforwardly without extensive further computations. The metabolic state, together with a set of Michaelis constants or saturation parameters, usually uniquely specifies the set of enzyme-kinetic parameters.

where $\theta$ denotes the logarithmic partial derivative of $r_{2}(S)$ with respect to its substrate $S$. We obtain

$$
\theta:=\left.\frac{\partial \ln r}{\partial \ln S}\right|_{S^{0}}=\frac{1}{1+\frac{S^{0}}{K_{M}}} \in[0,1] .
$$

Clearly, the value of $\theta \in[0,1]$ is confined to a well defined interval that describes the degree of saturation of the reaction $r$ with respect to its substrate $S$. For $S^{0}<<K_{M}$ the reaction operates in the linear regime with $\theta \approx 1$, whereas for $S^{0} \gg K_{M}$ the reaction operates in the saturated regime, with $\theta \approx 0$. A similar reasoning applies to almost any arbitrary functional form of a biochemical rate equation. Consequently, the saturation parameters $Q_{i j}$ can often be sampled directly within well-defined intervals, without referring to any explicit functional form of the rate equations $[48,59,88]$. Once each element of the matrix $\mathbf{Q}$ is assigned, the Jacobian is specified according to Eq. (8). For the example discussed above, we obtain

$$
\mathbf{J}=-\frac{r^{0}}{S^{0}} \theta .
$$

For a detailed evaluation, including more complex examples, see Steuer and Junker [20].

It should be emphasized that for most complex rate equations, the matrix $\mathbf{Q}$ can be split up according to contributions from kinetics, regulation and thermodynamics [20]. In particular, a large number of rate equations can be written as a product similar to Eq. (3)

$$
r=f_{\text {reg }} \cdot r_{\text {kin }}^{+} \cdot f_{\text {thd }},
$$

with $f_{\text {reg }}$ denoting a regulatory factor, $r_{\text {kin }}^{+}$denoting a kinetic factor, and $f_{\text {thd }}=1-\Gamma / K_{\mathrm{eq}}$ denoting the thermodynamic factor. In this case, the matrix $\mathbf{Q}$ is given as the sum of the individual contributions

$$
\mathbf{Q}=\mathbf{Q}_{\mathrm{reg}}+\mathbf{Q}_{\mathrm{kin}}+\mathbf{Q}_{\mathrm{thd}}
$$

such that the elements of each matrix can be assigned to well-defined and biologically reasonable intervals. We note that our top-down modeling strategy does not entail any approximation or simplification of the system. Rather it draws upon a description in terms of a generalized set of parameters. On the one hand, we have a description of the metabolic network in terms of the metabolic state $\left\{\mathbf{r}^{0}, \mathbf{S}^{\mathbf{0}}\right\}$, the affinity constants $\mathbf{K}_{M}$ (or, when applicable, the saturation constant), as well as the equilibrium constants $\mathbf{K}_{\mathrm{eq}}$. On the other hand, we have a description in terms of the classic enzyme-kinetic parameters $\mathbf{K}_{M}, \mathbf{V}_{M}$, and $\mathbf{K}_{\mathrm{eq}}$. Both sets of parameters correspond to equivalent descriptions and are, at least in principle, interconvertible, as illustrated in Fig. (4).

The approach describe here was recently applied to a number of metabolic systems of reasonable complexity, including an analysis of the metabolism of human erythrocytes [53]. These early results indicate that distinct metabolic states are indeed associated with unique dynamic properties. In particular, the results, together with results obtained by similar strategies $[49,54,56,59,60]$ hold the promise that a detailed understanding of large-scale system does not necessarily require comprehensive knowledge of all kinetic parameters. Rather, a combination of phenotypic data, large-scale measurements, heuristic assumptions about typical rate equations, together with appropriate numerical schemes, allows for a fast and efficient way to explore the properties of large-scale metabolic networks. In this sense, Monte Carlo methods provide a path towards the construction and evaluation of large-scale kinetic models of cellular metabolism in the face of uncertain and only partially available kinetic data. 


\section{DISCUSSION AND CONCLUSIONS}

Computational modeling has become an indispensable tool to understand the function and dynamics of biochemical networks and is increasingly recognized as a credible contribution to molecular biology. As put by Mogilner et al. [89], "the closer the complete systematic understanding of cell behavior is, the more important modeling becomes and the more experiment and theory merge". In this respect, great strides have been made to facilitate computational modeling also beyond the small group of researchers that are traditionally associated with theoretical and computational biology. Several trends can be observed in the literature: First, the community strives for a unified nomenclature to describe computational models. The systems biology markup language (SBML) is emerging as a quasi-standard description format for biochemical reaction networks [90]. Second, minimal quality and reporting standards for the encoding and annotation of computational models are defined and increasingly adopted by the community [91]. Importantly, a standard description format, together with minimal reporting standards, facilitates the exchange and reuse of models using model repositories [92, 93], as well as the development of automated tools and software platforms to construct, handle, visualize, and interrogate computational models [94, 95]. In particular, a considerable number of software applications have become available that also enable users without extensive experience in programming to efficiently implement kinetic models of cellular processes [96-100].

However, the challenges ahead clearly go beyond nomenclature and model repositories. Detailed large-scale models of biochemical processes are still scarce - a fact that reflects the considerable difficulties associated with their construction. Arguably most advances have been made with respect to metabolic networks, where the reconstruction of the stoichiometry is considered to be almost comprehensive, at least for a small number of model species [58]. Nonetheless, even within the rather confined realm of central metabolism as yet unknown reaction paths continuously emerge and promiscuous enzymes might play a much larger role that currently anticipated [101, 102]. Likewise, further steps must aim to integrate multiple levels of cellular regulation, incorporating gene expression, enzyme concentrations and activities, together with cell metabolism within a single computational model [103-105]. In this respect, of particular interest is the interplay between a circadian clock and metabolism [106, 107], as well as other cellular and metabolic cycles [108-112].

Going beyond the stoichiometry, a great challenge is the transition from a static topological to a dynamic description of cellular pathways and mechanisms. The construction of kinetic models requires detailed knowledge of the underlying processes, supplemented by quantitative data of sufficient accuracy - requirements that are currently only rarely met in practice. Nonetheless, kinetic models are probably the ultimate stepping stone to truly understand the dynamic nature of cellular regulation. As yet, the functional consequences of regulatory interactions is, despite (or because of) the success of stoichiometric analysis, almost entirely uncharted territory. While for small systems and individual pathways, the relevance of dynamic properties and metabolic regulation is increasingly apparent, the consequences of autocatalytic pathways, dynamic instabilities, and feedback regulation for the function of large-scale networks is only rarely discussed [53, 113-121]. In this sense, the open questions amenable to a computational description are manifold - and often relate to the foundations of metabolic regulation and function [122124]. Computational modeling must not only aim to reproduce an observed time-course or response in silico, but must - and does already - contribute to formulate the fundamental paradigms in our interpretation of cellular functions.

\section{ACKNOWLEDGEMENTS}

RS is supported by the systems biology initiative "FORSYS-Partner: Systems Biology of Cyanobacterial Biofuel Production" funded German Federal Ministry of Education and Research (BMBF), grant no. 0315274B; and SysMO, a European transnational funding and research initiative on "Systems Biology of Microorganisms", grant nos. BBF0035361 (SulfoSYS), BBF0035521 (SysMOLAB), and BBF0035281 (MOSES).

\section{REFERENCES}

[1] A. Goldbeter, "Computational approaches to cellular rhythms", Nature, vol. 420, pp. 238-245, November 2002.

[2] J. Jaeger, S. Surkova, M. Blagov, H. Janssens, D. Kosman, K. N. Kozlov, Manu, E. Myasnikova, C. E. Vanario-Alonso, M. Samsonova, D. H. Sharp, and J. Reinitz, "Dynamic control of positional information in the early Drosophila embryo", Nature, vol. 430, pp. 368-371, July 2004.

[3] H. Meinhardt, "Computational modelling of epithelial patterning", Curr. Opin. Genet. Dev., vol. 17, pp. 272-280, August 2007.

[4] R. Heinrich, B. G. Neel, and T. A. Rapoport, "Mathematical models of protein kinase signal transduction", Mol. Cell., vol. 9, pp. 957-970, May 2002.

[5] D. B. Kell, "The virtual human: towards a global systems biology of multiscale, distributed biochemical network models", IUBMB Life, vol. 59, pp. 689-695, November 2007.

[6] R. Steuer, "Computational approaches to the topology, stability and dynamics of metabolic networks", Phytochemistry, vol. 68, no. 1618, pp. 2139-2151, 2007.

[7] N. Jamshidi, and B. Ø. Palsson, "Formulating genome-scale kinetic models in the post-genome era", Mol. Syst. Biol., vol. 4, pp.171, 2008.

[8] E. Pieroni, S. de la Fuente van Bentem, G. Mancosu, E. Capobianco, H. Hirt, and A. de la Fuente, "Protein networking: insights into global functional organization of proteomes", Proteomics, vol. 8, pp. 799-816, February 2008.

[9] J. Schellenberger, and B. Ø. Palsson, "Use of randomized sampling for analysis of metabolic networks", J. Biol. Chem., vol. 284, pp. 5457-5461, February 2009.

[10] H. V. Westerhoff, A. Kolodkin, R. Conradie, S. J. Wilkinson, F. J. Bruggeman, K. Krab, J. H. van Schuppen, H. Hardin, B. M. Bakker, M. J. Mon, K. N. Rybakova, M. Eijken, H. J. P. van Leeuwen, and J. L. Snoep, "Systems biology towards life in silico: mathematics of the control of living cells", J. Math. Biol., vol. 58, pp. 7-34, January 2009.

[11] B. Chance, "The kinetics of the enzyme-substrate compound of peroxidase 1943”, Adv. Enzymol. Relat. Areas Mol. Biol., vol. 73, pp. 3-23, 1999.

[12] D. Garfinkel, L. Garfinkel, M. Pring, S. B. Green, and B. Chance, "Computer applications to biochemical kinetics", Аппи. Rev. Biochem., vol. 39, pp. 473-498, 1970.

[13] D. Garfinkel, "Simulating biochemical activity in physiological systems," Simulation, vol. 28, pp. 193-196, 1977.

[14] H. Kitano, "Computational systems biology", Nature, vol. 420, pp. 206-210, November 2002.

[15] J. E. Bailey, "Mathematical modeling and analysis in biochemical engineering: past accomplishments and future opportunities", Biotechnol. Prog., vol. 14, no. 1, pp. 8-20, 1998. 
[16] R. N. Gutenkunst, J. J. Waterfall, F. P. Casey, K. S. Brown, C. R. Myers, and J. P. Sethna, "Universally sloppy parameter sensitivities in systems biology models", PLoS Comput. Biol., vol. 3, pp. 18711878, Octuber 2007.

[17] J. L. Casti, Would-be-worlds: How Simulation Is Changing the Frontiers of Science. John Wiley \& Sons Inc, 1998.

[18] Y. Lazebnik, "Can a biologist fix a radio?-or, what I learned while studying apoptosis" Cancer Cell., vol. 2, pp. 179-182, September 2002.

[19] J. J. Tyson, K. C. Chen, and B. Novak, "Sniffers, buzzers, toggles and blinkers: dynamics of regulatory and signaling pathways in the cell”, Curr. Opin. Cell. Biol., vol. 15, pp. 221-231, 2003.

[20] R. Steuer, and B. H. Junker, "Computational models of metabolism: Stability and regulation in metabolic networks," Adv. Chem. Phys., vol. 142, pp. 105-251, 2009.

[21] M. A. Savageau, "Biochemical systems analysis. i. Some mathematical properties of the rate law for the component enzymatic reactions", J. Theor. Biol., vol. 25, pp. 365-369, December 1969.

[22] M. A. Savageau, "Biochemical systems analysis. ii. the Steadystate solutions for an n-pool system using a power-law approximation”, J. Theor. Biol., vol. 25, pp. 370-379, December 1969.

[23] H. Kacser, and J. A. Burns, "The control of flux," Symp. Soc. Exp. Biol., vol. 27, pp. 65-104, 1973.

[24] R. Heinrich, and T. A. Rapoport, "A linear steady-state treatment of enzymatic chains. General properties, control and effector strength”, Eur. J. Biochem., vol. 42, pp. 89-95, February 1974.

[25] A.-L. Barab'asi, and Z. N. Oltvai, "Network biology: understanding the cell's functional organization", Nat. Rev. Genet., vol. 5, pp. 101-113, February 2004.

[26] S. Schuster, A. von Kamp, and M. Pachkov, "Understanding the roadmap of metabolism by pathway analysis" Methods Mol. Biol., vol. 358, pp. 199-226, 2007.

[27] B.Ø. Palsson, "Metabolic systems biology", FEBS Lett., vol. 583, pp. 3900-3904, September 2009.

[28] H. Knoop, Y. Zilliges,W. Lockau, and R. Steuer, "The metabolic network of Synechocystis sp. PCC 6803: Systemic properties of autotrophic growth", Plant Physiol., vol. 154, pp. 410-422, July 2010.

[29] S. A. Kauffman, "Metabolic stability and epigenesis in randomly constructed genetic nets" J. Theor. Biol., vol. 22, pp. 437-467, March 1969.

[30] S. Bornholdt, "Boolean network models of cellular regulation: prospects and limitations", J. R. Soc. Interface, vol. 5 (Suppl. 1), pp. S85-S94, August 2008.

[31] B. N. Kholodenko, "Cell-signalling dynamics in time and space", Nat. Rev. Mol. Cell. Biol., vol. 7, pp. 165-176, March 2006.

[32] H. H. McAdams, and A. Arkin, "It's a noisy business! Genetic regulation at the nanomolar scale", Trends Genet., vol. 15, pp. 6569, February 1999.

[33] W. J. Blake, M. Kaern, C. R. Cantor, and J. J. Collins, "Noise in eukaryotic gene expression", Nature, vol. 422, pp. 633-637, April 2003.

[34] N. Maheshri, and E. K. O'Shea, "Living with noisy genes: How cells function reliably with inherent variability in gene expression", Annu. Rev. Biophys. Biomol. Struct., vol. 36, pp. 413-434, 2007.

[35] A. Sackmann, M. Heiner, and I. Koch, "Application of petri net based analysis techniques to signal transduction pathways", $B M C$ Bioinformatics, vol. 7, pp. 482, 2006.

[36] R. Breitling, D. Gilbert, M. Heiner, and R. Orton, "A structured approach for the engineering of biochemical network models, illustrated for signalling pathways" Brief Bioinform., vol. 9, pp. 404421, September 2008.

[37] P. Mendes, and D. Kell, "Non-linear optimization of biochemical pathways: applications to metabolic engineering and parameter estimation", Bioinformatics, vol. 14, no. 10, pp. 869-883, 1998.

[38] I.-C. Chou, and E. O. Voit, "Recent developments in parameter estimation and structure identification of biochemical and genomic systems", Math. Biosci., vol. 219, pp. 57-83, June 2009.

[39] S. Kaplan, A. Bren, A. Zaslaver, E. Dekel, and U. Alon, "Diverse two-dimensional input functions control bacterial sugar genes", Mol. Cell., vol. 29, pp. 786-792, March 2008.

[40] R. Albert, "Network inference, analysis, and modeling in systems biology", Plant Cell, vol. 19, pp. 3327-3338, November 2007.

[41] S. M. Assmann, and R. Albert, "Discrete dynamic modeling with asynchronous update, or how to model complex systems in the ab- sence of quantitative information" Methods Mol. Biol., vol. 553, pp. 207-225, 2009.

[42] T. Gowda, S. Vrudhula, and S. Kim, "Modeling of gene regulatory network dynamics using threshold logic", Ann. N Y Acad. Sci., vol. 1158, pp. 71-81, March 2009.

[43] F. Horn, and R. Jackson, "General mass action kinetics," Archiv. Ration. Mech. Anal., vol. 47, pp. 81-116, 1972.

[44] M. Feinberg, and F. J. M. Horn, "Dynamics of open chemical systems and the algebraic structure of the underlying reaction network," Chem. Eng. Sci., vol. 29, no. 3, pp. 775-787, 1974.

[45] G. Craciun, Y. Tang, and M. Feinberg, "Understanding bistability in complex enzyme-driven reaction networks," Proc. Natl. Acad. Sci. USA, vol. 103, pp. 8697-8702, June 2006.

[46] B. L. Clarke, "Stoichiometric network analysis", Cell. Biophys., vol. 12, pp. 237-253, 1988.

[47] W. Liebermeister, and E. Klipp, "Biochemical networks with uncertain parameters" Syst. Biol., (Stevenage), vol. 152, pp. 97-107, September 2005

[48] R. Steuer, T. Gross, J. Selbig, and B. Blasius, "Structural kinetic modeling of metabolic networks", Proc. Natl. Acad. Sci. USA, vol. 103, pp. 11868-11873, August 2006.

[49] W. Liebermeister, and E. Klipp, "Bringing metabolic networks to life: convenience rate law and thermodynamic constraints", Theor. Biol. Med. Model., vol. 3, p. 41, 2006.

[50] W. Liebermeister and E. Klipp, "Bringing metabolic networks to life: integration of kinetic, metabolic, and proteomic data", Theor. Biol. Med. Model., vol. 3, p. 42, 2006.

[51] R. Steuer, A. N. Nesi, A. R. Fernie, T. Gross, B. Blasius, and J. Selbig, "From structure to dynamics of metabolic pathways: application to the plant mitochondrial TCA cycle", Bioinformatics, vol. 23, pp. 1378-1385, June 2007.

[52] L. Kuepfer, M. Peter, U. Sauer, and J. Stelling, "Ensemble modeling for analysis of cell signaling dynamics", Nat. Biotechnol., vol. 25, pp. 1001-1006, September 2007.

[53] S. Grimbs, J. Selbig, S. Bulik, H.-G. Holzhtter, and R. Steuer, "The stability and robustness of metabolic states: identifying stabilizing sites in metabolic networks", Mol. Syst. Biol., vol. 3, p.146, 2007.

[54] L. M. Tran, M. L. Rizk, and J. C. Liao, "Ensemble modeling of metabolic networks", Biophys. J., vol. 95, pp. 5606-5617, December 2008.

[55] J. Schaber, W. Liebermeister, and E. Klipp, "Nested uncertainties in biochemical models", IET Syst. Biol., vol. 3, pp. 1-9, January 2009.

[56] H.-M. Kaltenbach, S. Dimopoulos, and J. Stelling, "Systems analysis of cellular networks under uncertainty" FEBS Lett., vol. 583 pp. 3923-3930, October 2009.

[57] J. Stelling, S. Klamt, K. Bettenbrock, S. Schuster, and E. D. Gilles, "Metabolic network structure determines key aspects of functionality and regulation", Nature, vol. 420, pp. 190-193, November 2002.

[58] A. M. Feist, M. J. Herrg ${ }^{\circ}$ ard, I. Thiele, J. L. Reed, and B. Ø. Palsson, "Reconstruction of biochemical networks in microorganisms", Nat. Rev. Microbiol., vol. 7, pp. 129-143, February 2009.

[59] L. Wang, I. Birol, and V. Hatzimanikatis, "Metabolic control analysis under uncertainty: framework development and case studies", Biophys. J., vol. 87, pp. 3750-3763, December 2004.

[60] K. Smallbone, E. Simeonidis, D. S. Broomhead, and D. B. Kell, "Something from nothing: bridging the gap between constraintbased and kinetic modelling", FEBS J., vol. 274, pp. 5576-5585, November 2007.

[61] D. A. Adiamah, J. Handl, and J.-M. Schwartz, "Streamlining the construction of large-scale dynamic models using generic kinetic equations", Bioinformatics, vol. 26, pp. 1324-1331, May 2010.

[62] J. M. Rohwer, A. J. Hanekom, C. Crous, J. L. Snoep, and J. H. S. Hofmeyr, "Evaluation of a simplified generic bi-substrate rate equation for computational systems biology", Syst. Biol.,(Stevenage), vol. 153, pp. 338-341, September 2006.

[63] S. Bulik, S. Grimbs, C. Huthmacher, J. Selbig, and H. G. Holzhtter, "Kinetic hybrid models composed of mechanistic and simplified enzymatic rate laws-a promising method for speeding up the kinetic modelling of complex metabolic networks", FEBS J., vol. 276, pp. 410-424, January 2009.

[64] F. Hadlich, S. Noack, and W. Wiechert, "Translating biochemical network models between different kinetic formats", Metab. Eng., vol. 11, pp. 87-100, March 2009.

[65] A. Dr"ager, M. Kronfeld, M. J. Ziller, J. Supper, H. Planatscher, J. B. Magnus, M. Oldiges,O. Kohlbacher, and A. Zell, "Modeling 
metabolic networks in C. glutamicum: a comparison of rate laws in combination with various parameter optimization strategies", $B M C$ Syst. Biol., vol. 3, p. 5, 2009.

[66] W. Liebermeister, J. Uhlendorf, and E. Klipp, "Modular rate laws for enzymatic reactions: thermodynamics, elasticities and implementation" Bioinformatics, vol. 26, pp. 1528-1534, June 2010.

[67] R. A. Alberty, Thermodynamics of Biochemical Reactions. New York: John Wiley \& Sons, Inc., 2003.

[68] R. N. Goldberg, Y. B. Tewari, and T. N. Bhat, "Thermodynamics of enzyme-catalyzed reactions-a database for quantitative biochemistry", Bioinformatics, vol. 20, pp. 2874-2877, November 2004

[69] C. S. Henry, M. D. Jankowski, L. J. Broadbelt, and V. Hatzimanikatis, "Genome-scale thermodynamic analysis of Escherichia coli metabolism”, Biophys. J., vol. 90, pp. 1453-1461, February 2006.

[70] A. M. Feist, C. S. Henry, J. L. Reed, M. Krummenacker, A. R. Joyce, P. D. Karp, L. J. Broadbelt, V. Hatzimanikatis, and B. Ø. Palsson, "A genome-scale metabolic reconstruction for Escherichia coli $\mathrm{k}-12 \mathrm{mg} 1655$ that accounts for 1260 ORFs and thermodynamic information", Mol. Syst. Biol., vol. 3, p. 121, 2007.

[71] M. D. Jankowski, C. S. Henry, L. J. Broadbelt, and V. Hatzimanikatis, "Group contribution method for thermodynamic analysis of complex metabolic networks", Biophys. J., vol. 95, pp. 1487-1499, August 2008.

[72] I. Rojas, M. Golebiewski, R. Kania, O. Krebs, S. Mir, A. Weidemann, and U. Wittig, "Storing and annotating of kinetic data", In Silico. Biol., vol. 7, no. 2, pp. S37-S44, 2007.

[73] A. Chang, M. Scheer, A. Grote, I. Schomburg, and D. Schomburg, "BRENDA, AMENDA and FRENDA the enzyme information system: new content and tools in 2009" , Nucleic Acids Res., vol. 37, pp. D588-D592, January 2009.

[74] I. Spasic, E. Simeonidis, H. L. Messiha, N. W. Paton, and D. B. Kell, "Kipar, a tool for systematic information retrieval regarding parameters for kinetic modelling of yeast metabolic pathways", Bioinformatics, vol. 25, pp. 1404-1411, June 2009.

[75] Y. Gibon, O. E. Blaesing, J. Hannemann, P. Carillo, M. H“ohne, J. H. M. Hendriks, N. Palacios, J. Cross, J. Selbig, and M. Stitt, "A robot-based platform to measure multiple enzyme activities in Arabidopsis using a set of cycling assays: comparison of changes of enzyme activities and transcript levels during diurnal cycles and in prolonged darkness", Plant Cell, vol. 16, pp. 3304-3325, December 2004

[76] S. Borger, W. Liebermeister, J. Uhlendorf, and E. Klipp, "Automatically generated model of a metabolic network", Genome Inform, vol. 18, pp. 215-224, 2007.

[77] B. H. Junker, J. Lonien, L. E. Heady, A. Rogers, and J. Schwender, "Parallel determination of enzyme activities and in vivo fluxes in Brassica napus embryos grown on organic or inorganic nitrogen source", Phytochemistry, vol. 68, no. 16-18, pp. 2232-2242, 2007.

[78] S. Sahle, P. Mendes, S. Hoops, and U. Kummer, "A new strategy for assessing sensitivities in biochemical models" Philos. Transact. A. Math. Phys. Eng. Sci., vol. 366, pp. 3619-3631, October 2008.

[79] O. Fiehn, "Combining genomics, metabolome analysis, and biochemical modelling to understand metabolic networks", Comp. Funct. Genomics, vol. 2, no. 3, pp. 155-168, 2001.

[80] D. B. Kell, "Metabolomics and systems biology: making sense of the soup", Curr. Opin. Microbiol., vol. 7, pp. 296-307, June 2004.

[81] R. Goodacre, S. Vaidyanathan, W. B. Dunn, G. G. Harrigan, and D. B. Kell, "Metabolomics by numbers: acquiring and understanding global metabolite data", Trends Biotechnol., vol. 22, pp. 245-252, May 2004

[82] U. Sauer, "Metabolic networks in motion: 13C-based flux analysis", Mol. Syst. Biol., vol. 2, p. 62, 2006.

[83] N. Zamboni, and U. Sauer, "Novel biological insights through metabolomics and 13c-flux analysis", Curr. Opin. Microbiol., vol. 12, pp. 553-558, October 2009.

[84] N. Zamboni, S.-M. Fendt, M. Rhl, and U. Sauer, "(13) C-based metabolic flux analysis", Nat. Protoc., vol. 4, no. 6, pp. 878-892, 2009.

[85] N. J. Kruger, and R. G. Ratcliffe, "Insights into plant metabolic networks from steady-state metabolic flux analysis", Biochimie, vol. 91, pp. 697-702, June 2009.

[86] N. Ishii, K. Nakahigashi, T. Baba, M. Robert, T. Soga, A. Kanai, T. Hirasawa, M. Naba, K. Hirai, A. Hoque, P. Y. Ho, Y. Kakazu, K. Sugawara, S. Igarashi, S. Harada, T. Masuda, N. Sugiyama, T. Togashi, M. Hasegawa, Y. Takai, K. Yugi, K. Arakawa, N. Iwata, Y. Toya, Y. Nakayama, T. Nishioka, K. Shimizu, H. Mori, and M.
Tomita, "Multiple high-throughput analyses monitor the response of E. coli to perturbations", Science, vol. 316, pp. 593-597, April 2007.

[87] B. D. Bennett, E. H. Kimball, M. Gao, R. Osterhout, S. J. V. Dien, and J. D. Rabinowitz, "Absolute metabolite concentrations and implied enzyme active site occupancy in Escherichia coli", Nat. Chem. Biol., vol. 5, pp. 593-599, August 2009.

[88] T. Gross and U. Feudel, "Generalized models as a universal approach to the analysis of nonlinear dynamical systems", Phys. Rev. E. Stat. Nonlin. Soft. Matter. Phys., vol. 73, p. 016205, January 2006.

[89] A. Mogilner, R. Wollman, and W. F. Marshall, "Quantitative modeling in cell biology: what is it good for?," Dev. Cell., vol. 11, pp. 279-287, September 2006.

[90] M. Hucka, A. Finney, H. M. Sauro, H. Bolouri, J. C. Doyle, H. Kitano, A. P. Arkin, B. J. Bornstein, D. Bray, A. Cornish-Bowden, A. A. Cuellar, S. Dronov, E. D. Gilles, M. Ginkel, V. Gor, I. I. Goryanin, W. J. Hedley, T. C. Hodgman, J.-H. Hofmeyr, P. J. Hunter, N. S. Juty, J. L. Kasberger, A. Kremling, U. Kummer, N. L. Nov`ere, L. M. Loew, D. Lucio, P. Mendes, E. Minch, E. D. Mjolsness, Y. Nakayama, M. R. Nelson, P. F. Nielsen, T. Sakurada, J. C. Schaff, B. E. Shapiro, T. S. Shimizu, H. D. Spence, J. Stelling, K. Takahashi, M. Tomita, J. Wagner, J. Wang, and S. B. M. L. Forum, "The systems biology markup language (SBML): a medium for representation and exchange of biochemical network models", Bioinformatics, vol. 19, pp. 524-531, March 2003.

[91] N. L. Novere, A. Finney, M. Hucka, U. S. Bhalla, F. Campagne, J. Collado-Vides, E. J. Crampin, M. Halstead, E. Klipp, P. Mendes, P. Nielsen, H. Sauro, B. Shapiro, J. L. Snoep, H. D. Spence, and B. L. Wanner, "Minimum information requested in the annotation of biochemical models (MIRIAM)", Nat. Biotechnol., vol. 23, pp. 1509-1515, December 2005.

[92] N. L. Novre, B. Bornstein, A. Broicher, M. Courtot, M. Donizelli, H. Dharuri, L. Li, H. Sauro, M. Schilstra, B. Shapiro, J. L. Snoep, and M. Hucka, "Biomodels database: a free, centralized database of curated, published, quantitative kinetic models of biochemical and cellular systems", Nucleic Acids Res., vol. 34, pp. D689-D691, January 2006.

[93] C. van Gend, and J. L. Snoep, "Systems biology model databases and resources", Essays Biochem., vol. 45, pp. 223-236, 2008.

[94] H.M. Sauro, and F. T. Bergmann, "Standards and ontologies in computational systems biology" Essays Biochem., vol. 45, pp. 211 222, 2008.

[95] L. Endler, N. Rodriguez, N. Juty, V. Chelliah, C. Laibe, C. Li, and N. L. Novre, "Designing and encoding models for synthetic biology", J. R. Soc. Interface, vol. 6, (Suppl. 4), pp. S405-S417, August 2009.

[96] B. G. Olivier, and J. L. Snoep, "Web-based kinetic modelling using JWS online”, Bioinformatics, vol. 20, pp. 2143-2144, September 2004.

[97] S. Hoops, S. Sahle, R. Gauges, C. Lee, J. Pahle, N. Simus, M. Singhal, L. Xu, P. Mendes, and U. Kummer, "Copasi-a complex pathway simulator", Bioinformatics, vol. 22, pp. 3067-3074, December 2006.

[98] P.Mendes, S. Hoops, S. Sahle, R. Gauges, J. Dada, and U. Kummer, "Computational modeling of biochemical networks using Copasi”, Methods Mol. Biol., vol. 500, pp. 17-59, 2009.

[99] A. Weidemann, S. Richter, M. Stein, S. Sahle, R. Gauges, R. Gabdoulline, I. Surovtsova, N. Semmelrock, B. Besson, I. Rojas, R. Wade, and U. Kummer, "Sycamore-a systems biology computational analysis and modeling research environment", Bioinformatics, vol. 24, pp. 1463-1464, June 2008.

[100] S. Mirschel, K. Steinmetz, M. Rempel, M. Ginkel, and E. D. Gilles, "PROMOT: modular modeling for systems biology", Bioinformatics, vol. 25, pp. 687-689, March 2009

[101] M. Arita, "The metabolic world of Escherichia coli is not small", Proc. Natl. Acad. Sci. USA, vol. 101, pp. 1543-1547, February 2004.

[102] K. Nakahigashi, Y. Toya, N. Ishii, T. Soga, M. Hasegawa, H. Watanabe, Y. Takai, M. Honma, H. Mori, and M. Tomita, "Systematic phenome analysis of escherichia coli multiple-knockout mutants reveals hidden reactions in central carbon metabolism", Mol. Syst. Biol., vol. 5, p. 306, 2009.

[103] A. de la Fuente and P.Mendes, "Integrative modelling of gene expression and cell metabolism", Appl. Bioinformatics, vol. 2, no. 2, pp. 79-90, 2003. 
[104] E. Klipp, B. Nordlander, R. Kruger, P. Gennemark, and S. Hohmann, "Integrative model of the response of yeast to osmotic shock", Nat. Biotechnol., vol. 23, pp. 975-982, August 2005.

[105] M. W. Covert, N. Xiao, T. J. Chen, and J. R. Karr, "Integrating metabolic, transcriptional regulatory and signal transduction models in Escherichia coli", Bioinformatics, vol. 24, pp. 2044-2050, September 2008.

[106] J. Kovac, J. Husse, and H. Oster, "A time to fast, a time to feast: the crosstalk between metabolism and the circadian clock", Mol. Cells, vol. 28, pp. 75-80, August 2009.

[107] C. B. Green, J. S. Takahashi, and J. Bass, "The meter of metabolism", Cell, vol. 134, pp. 728-742, September 2008.

[108] R. R. Klevecz, J. Bolen, G. Forrest, and D. B. Murray, "A genomewide oscillation in transcription gates DNA replication and cell cycle", Proc. Natl. Acad. Sci. USA, vol. 101, pp. 1200-1205, February 2004.

[109] D. Lloyd, and D. B. Murray, "The temporal architecture of eukaryotic growth”, FEBS Lett., vol. 580, pp. 2830-2835, May 2006.

[110] B. P. Tu, and S. L. McKnight, "Metabolic cycles as anderlying basis of biological oscillations", Nat. Rev. Mol. Cell. Biol., vol. 7, pp. 696-701, September 2006.

[111] D. B. Murray, M. Beckmann, and H. Kitano, "Regulation of yeast oscillatory dynamics", Proc. Natl. Acad. Sci. USA, vol. 104, pp. 2241-2246, February 2007.

[112] B. P. Tu, R. E. Mohler, J. C. Liu, K. M. Dombek, E. T. Young, R. E. Synovec, and S. L. McKnight, "Cyclic changes in metabolic state during the life of a yeast cell", Proc. Natl. Acad. Sci. USA, vol. 104, pp. 16886-16891, October 2007.

[113] B. Teusink, M. C. Walsh, K. van Dam, and H. V. Westerhoff, "The danger of metabolic pathways with turbo design", Trends Biochem. Sci., vol. 23, pp. 162-169, May 1998.

[114] R. Scheibe, J. E. Backhausen, V. Emmerlich, and S. Holtgrefe, "Strategies to maintain redox homeostasis during photosynthesis under changing conditions", J. Exp. Bot., vol. 56, pp. 1481-1489, June 2005.
[115] R. J. Prill, P. A. Iglesias, and A. Levchenko, "Dynamic properties of network motifs contribute to biological network organization", PLoS Biol., vol. 3, p. e343, November 2005.

[116] E. Voit, A. R. Neves, and H. Santos, "The intricate side of systems biology", Proc. Natl. Acad. Sci. USA, vol. 103, pp. 9452-9457, June 2006.

[117] A. Ma'ayan, G. A. Cecchi, J. Wagner, A. R. Rao, R. Iyengar, and G. Stolovitzky, "Ordered cyclic motifs contribute to dynamic stability in biological and engineered networks", Proc. Natl. Acad. Sci. USA, vol. 105, pp. 19235-19240, December 2008.

[118] J. R. Haanstra, A. van Tuijl, P. Kessler, W. Reijnders, P. A. M. Michels, H. V. Westerhoff, M. Parsons, and B. M. Bakker, "Compartmentation prevents a lethal turbo-explosion of glycolysis in trypanosomes", Proc. Natl. Acad. Sci. USA, vol. 105, pp. 1771817723, November 2008.

[119] P. Morandini, "Rethinking metabolic control," Plant Sci., vol. 176, pp. 441-451, 2009.

[120] V. Baldazzi, D. Ropers, Y. Markowicz, D. Kahn, J. Geiselmann, and H. de Jong, "The carbon assimilation network in Escherichia coli is densely connected and largely sign-determined by directions of metabolic fluxes", PLoS Comput. Biol., vol. 6, no. 6, p. e1000812, 2010.

[121] S. Goyal, J. Yuan, T. Chen, J. D. Rabinowitz, and N. S. Wingreen, "Achieving optimal growth through product feedback inhibition in metabolism”, PLoS Comput. Biol., vol. 6, no. 6, p. e1000802, 2010.

[122] T. Pfeiffer, S. Schuster, and S. Bonhoeffer, "Cooperation and competition in the evolution of ATP-producing pathways", Science, vol. 292, pp. 504-507, April 2001.

[123] B. Papp, B. Teusink, and R. A. Notebaart, "A critical view of metabolic network adaptations", HFSP J., vol. 3, pp. 24-35, February 2009.

[124] D. Molenaar, R. van Berlo, D. de Ridder, and B. Teusink, "Shifts in growth strategies reflect tradeoffs in cellular economics", Mol. Syst. Biol., vol. 5, p. 323, 2009.

(C) Ralf Steuer; Licensee Bentham Open.

This is an open access article licensed under the terms of the Creative Commons Attribution Non-Commercial License (http://creativecommons.org/licenses/by-nc/3.0/) which permits unrestricted, non-commercial use, distribution and reproduction in any medium, provided the work is properly cited. 